\title{
SIKHS EN BARCELONA. CARACTERÍSTICAS, IDIOSINCRASIA Y CAUSALIDADES DE LA CREACIÓN Y ESTABLECIMIENTO DE UNA COMUNIDAD EN EL MARCO GLOBAL DE LAS MIGRACIONES TRANSNACIONALES*
}

\author{
SIKHS IN BARCELONA. CHARACTERISTICS, IDIOSYNCRASY AND THE \\ CAUSALITIES IN THE CREATION AND THE SETTLEMENT OF A COMMUNITY \\ IN THE GLOBAL FRAME OF TRANSNATIONAL MIGRATIONS
}

\author{
Sandra Santos-Fraile ${ }^{1}$ \\ Universidad Complutense de Madrid. Departamento de Antropología Social y Psicología Social
}

Recibido: 5 de noviembre de 2019; Aprobado: 22 de julio de 2020.

Cómo citar este artículo / Citation: Santos-Fraile, Sandra. 2021. "Sikhs en Barcelona. Características, idiosincrasia y causalidades de la creación y establecimiento de una comunidad en el marco global de las migraciones transnacionales". Disparidades. Revista de Antropología 76(2): e017. doi: <https://doi.org/10.3989/dra.2021.017>.

RESUMEN: Si bien existen sikhs que habitan en diferentes lugares de España, en Barcelona se encuentra la comunidad más antigua, estable y probablemente organizada del país. El hecho de que los sikhs hayan inmigrado a España responde a razones que tienen que ver tanto con su bagaje histórico y su idiosincrasia como con fenómenos tan amplios como la globalización y los flujos globales transnacionales. Este trabajo trata de visibilizar la comunidad sikh de Barcelona -así como la existencia de otros sikhs en el Estado español- describiendo y explicando cómo y por qué vinieron hasta aquí y cuál es la forma en que se organizan y funcionan a diferencia de como lo hacen en otros lugares. Para llevar a cabo esta investigación se ha llevado a cabo trabajo de campo etnográfico durante más de 18 meses mediante etnografía multisituada en lugares como Barcelona, Londres, Delhi y el Panyab además de una importante revisión bibliográfica.

PALABRAS CLAVE: Sikhs; Barcelona; Migración y Diáspora: Globalización y comunidades transnacionales.

ABSTRACT: There are Sikhs living in different parts of Spain, but the oldest, most stable and organized community is settled in Barcelona. The Sikhs immigrated to Spain because of a number of reasons such as their idiosyncrasy; their historical and cultural background; and also because of phenomena such as globalization and global and transnational fluxes. The main aim of this paper is to make the Sikh community of Barcelona visible - and also the Sikhs in other parts of Spain. I am going to describe and explain why and how the Sikhs arrive in Barcelona and how they organize and live here, taking into account the differences between other places of settlement. Therefore, I have done anthropological fieldwork for more than 18 months, using the multi-sited ethnography in places such as Barcelona, London, Delhi and the Punjab. I have also done an extensive review of published literature.

KEYWORDS: Sikhs; Barcelona; Migration and Diaspora; Globalization and Transnational Communities.

Copyright: @ 2021 CSIC. Este es un artículo de acceso abierto distribuido bajo los términos de la licencia de uso y distribución Creative Commons Reconocimiento 4.0 Internacional (CC BY 4.0).

\footnotetext{
* La investigación que ha llevado a este trabajo ha sido financiada parcialmente con una beca de Formación del Profesorado Universitario del Ministerio de Educación, Referencia: AP2005-1400.

1 Correo electrónico: sandrasf@ucm.es. ORCID iD: <https://orcid.org/0000-0002-2703-3000>.
} 


\section{INTRODUCCIÓN}

Sibien existen sikhs que habitan en diferentes lugares de España, esta investigación pone de manifiesto que en Barcelona se encuentra la comunidad más antigua, estable y organizada del país. Aunque si hay una característica que define la situación de los sikhs ${ }^{2}$ en España en general y en Barcelona en particular, en tanto que individuos pertenecientes a un grupo religioso con unas características idiosincrásicas propias y diferenciadas de otros hindúes ${ }^{3}$, e incluso de musulmanes, esta es su invisibilidad. Los sikhs pasan desapercibidos para la mayoría de los nativos, que los perciben como inmigrantes en general, pero que los confunden con otros hindúes o incluso con pakistaníes, obviando así su especificidad en tanto que grupo diferenciado. A pesar de ello, y desde que a finales de los años 70s y principios de los $80 \mathrm{~s}$ -según mis propios informantes-, los primeros sikhs se asentaran en Barcelona, la comunidad ha sufrido un crecimiento significativo, especialmente desde el año 2000.

Es indudable que el hecho de que no sea una de las comunidades más numerosas ni haya llevado a cabo demandas políticas o sociales con un efecto visibilizador de cara a la sociedad de acogida, hace que pase desapercibida y no reciba demasiada atención por parte de la población local; a excepción de cuando con motivo de sus celebraciones religiosas anuales hacen uso del espacio público con procesiones que lo convierten en un gurdwara transitorio y efímero (Santos-Fraile 2016) ${ }^{4}$. En cualquier caso, los sikhs

2 Aunque la Real Academia Española (RAE) en su edición más reciente, así como la mayoría de los medios de comunicación utilizan el vocablo sij y su derivado sijismo como transliteración del término sikh, por lo cuestionable de esta adaptación al castellano, he optado por mantener el uso de los términos sikh y sikhismo. Esta cuestión se discute más pormenorizadamente en mi tesis doctoral (Ver Santos-Fraile 2016).

3 A pesar de que en la RAE los términos hindú e hinduista en una de sus acepciones se consideran como sinónimos, en este trabajo, y a fin de distinguir claramente a quiénes nos estamos refiriendo en cada momento, se utilizará el término "hindús» para referirnos a aquellos/ as procedentes de la India, e "hinduistas" para las/los seguidores del hinduismo.

4 También su participación en el Forum Universal de las Culturas en 2004 en Barcelona puede considerarse un momento puntual de presentación de la comunidad y de conformación de su imagen frente a la sociedad de acogida. forman parte de la actual sociedad multicultural del Estado español, y el hecho de que los sikhs hayan inmigrado a este país responde a razones que tienen que ver tanto con su bagaje histórico y su idiosincrasia como con fenómenos tan amplios como la globalización y los flujos globales transnacionales.

Dado que no existen datos estadísticos o investigaciones al respecto, es difícil saber cuándo los primeros sikhs llegaron y se establecieron en el país. En cualquier caso, en la actualidad hay sikhs en Barcelona, Girona, Valencia, Madrid, Málaga, Murcia, Bilbao, Las Islas Canarias, Almería, Ceuta, y Melilla. No obstante, los casos de Ceuta y Melilla son bastante particulares por su condición de plazas fronterizas y su enclave en el norte de África. Los sikhs llegan allí después de procesos de inmigración irregular y permanecen hasta que encuentran el modo de continuar hacia provincias peninsulares.

Barcelona es el lugar donde se establece la mayor comunidad sikh y donde hay mayor número de gurdwaras -templos sikhs-. Existen gurdwaras en otras ciudades españolas pero resulta difícil saber más sobre los sikhs viviendo en otros lugares del Estado por la falta de trabajos de investigación al respecto.

Es precisamente esta la razón de la significancia del artículo que aquí se presenta. Si bien hay trabajos que ponen su punto de observación o análisis en lo conflictual de las sociedades multiculturales y el papel de las minorías en ellas (Herzog 2014), aquí vamos a mostrar cómo hay minorías que permanecen en base a disimilares estrategias de adaptación en función de los distintos contextos como ya señalaran Jacobsen y Myrvold (2014). Resulta, sin duda, este trabajo muy novedoso y de gran actualidad, en tanto que trata, por un lado, de visibilizar la existencia de sikhs en el Estado español, su forma de organización y la estructuración de sus comunidades -a partir del caso de Barcelona. También se describe y explica cómo y por qué vinieron hasta aquí y cuál es la forma en que se organizan y funcionan a diferencia de como lo hacen en otros lugares. Asimismo, se muestra cuáles son las interrelaciones y los vínculos que mantienen con otros sikhs en el marco de la diáspora y los flujos globales transnacionales, como parte del fenómeno de la globalización.

Considerando por tanto que 1) hay una significativa y según los datos estadísticos creciente población sikh en Barcelona y en otras provincias españolas; 
2) la inexistencia de estudios académicos previos específicos sobre este grupo en nuestro país; 3) que existe un gran desconocimiento sobre sus creencias, sus prácticas, sus formas de habitar y convivir así como sobre los vínculos e interconexiones que estos sikhs mantienen con otros correligionarios en la diáspora; y 4) sobre cómo se ubican los sikhs que habitan aquí con respecto a lo que Appadurai (2001) define como flujos culturales globales, el objetivo de este trabajo no es otro que visibilizar a esta comunidad sikh. Y lo hacemos incidiendo en las motivaciones y los medios que les han traído hasta aquí, en sus características en tanto que comunidad y en los procesos de negociación y los modos de asentamiento que llevan a cabo en Barcelona como lugar de llegada.

En primer lugar, se expone la metodología y técnicas de trabajo utilizadas. A continuación se presenta y contextualiza a la comunidad sikh de Barcelona en el marco de una comunidad sikh más amplia, transnacional y diaspórica. El tercer apartado muestra las características de la comunidad sikh de Barcelona, su proceso migratorio hasta la ciudad y las formas de llegada y asentamiento. Asimismo, se la contextualiza en el marco global. Finalmente, las conclusiones cierran el artículo.

\section{METOdología de TRABAJO}

En sus inicios, el diseño de la investigación planteaba como unidad de análisis al colectivo sikh de Barcelona, limitado a un ámbito geográfico (Barcelona). Pero los evidentes vínculos y conexiones transnacionales que forman parte de la realidad más cotidiana del grupo de estudio pronto mostró la necesidad de reformular este planteamiento, virando el diseño de la investigación hacia una etnografía multi-situada, donde Londres, Delhi y el Panyab se convertirían también en lugares clave para el desarrollo de este trabajo.

El trabajo de campo intensivo y continuado, con una duración de más de 18 meses $^{5}$, se inició llevando a cabo una aproximación etnográfica previa de la comunidad sikh de Barcelona, para lo que fue esencial la observación directa en los gurdwaras. Asimismo, tomar clases de panyabi fue uno de los

5 Aunque una vez finalizado el trabajo de campo, de forma interrumpida pero constante se ha mantenido contacto con los informantes principales. recursos que se planteó desde el inicio, aunque no se llegara a alcanzar la suficiente solvencia idiomática. Fundamental también para iniciar los primeros pasos en la investigación sería el contacto y la recogida de información en distintas instituciones que trabajan con los diferentes grupos religiosos, como la Asociación UNESCO para el Diálogo Interreligioso (UNESCOCAT), el Centro Interreligioso de Barcelona (CIB), Casa Asia, el Centro para el Estudio de las Tradiciones Religiosas ${ }^{6}$ o la Direcció General d'Afers Religiosos de la Generalitat de Catalunya. El contacto y la visita a todas estas instituciones, aunque bastante fructífera en cuanto a la consecución de los primeros contactos, pronto hizo evidente la ausencia de información relevante y firmes conocimientos sobre los sikhs en general y los sikhs en Barcelona o Cataluña en particular.

Aunque este trabajo se basa fundamentalmente en técnicas cualitativas, el acceso a los datos cuantitativos ha sido un recurso necesario con objeto de contextualizar la inmigración panyabi tanto en Barcelona como en Cataluña y en el resto del Estado Español. No obstante, los datos cuantitativos se han tomado con cierta cautela, pues como ya mostrara Nancy Scheper-Hughes (1999), los registros y datos estadísticos por razones muy diversas difícilmente se corresponden con la realidad de las situaciones que tratan de reflejar. El paso siguiente, y teniendo en cuenta el desconcocimiento generalizado sobre los sikhs y la falta de trabajos y recursos bibliográficos al alcance, fue la necesaria e intensiva consulta bibliográfica en bibliotecas de Londres y Delhi, entre ellas, la School of Oriental and African Studies (SOAS), así como en el Institute of Commonwealth Studies. En paralelo se inició el trabajo de campo en Southall (Londres), que sería continuado en diferentes periodos de tiempo con una duración total aproximada de siete meses, además de algunas estancias cortas complementarias. No obstante, el grueso del trabajo de campo se ha llevado a cabo de forma alternada en el Panyab y en Barcelona; siendo éste último lugar donde más tiempo se ha dedicado, comprendiendo prácticamente todo el período con excepción de los tiempos dedicados al resto de lugares. Asimismo, y dado que el trabajo de campo en la India fundamentalmente ha consistido en visitar y convivir con familias sikhs que habitan en Barcelona

6 En la actualidad, Centro de Estudio de las Tradiciones de Sabiduría (CETR). 
y que iban allí por vacaciones, negocios o para visitar a sus parientes, en ocasiones también Delhi ha sido lugar para el trabajo (así como otros estados de la India con ocasión de viajes cuando la movilidad de los sikhs a los que acompañaba o visitaba así lo requería). La duración total del trabajo de campo en la India ha sido de cuatro meses y medio, repartidos en dos estancias. Esta alternancia en los espacios para el trabajo de campo, así como convivir y acompañar en la India a los sikhs establecidos en Barcelona permitió estrechar contactos ya realizados, crear nuevos contactos y vínculos y, más importante si cabe para el propósito de esta investigación, poder establecer comparaciones. Es en este sentido, que para esta investigación una etnografía multisituada se presentaba como una necesidad desde el principio. Si bien George Marcus (1995: 80) señala que, a pesar de reconocerlas, esta metodología no se sustenta en conceptos o narrativas macroteóricas a la hora de enmarcar contextualmente a los sujetos de estudio, en nuestro caso se entiende que conceptos macroteóricos como la globalización, las diásporas e incluso las redes sí permiten contextualizar a los sujetos de estudio pero en procesos dinámicos transnacionales que no por estar espacialmente delimitados dejan de ser un marco adecuado para conocer, analizar y comprender individuos y prácticas, aunque deban entenderse de una manera más fluida que arraigados en un tiempo y espacios concretos.

Como Marcus (1995: 90) indica, la investigación multi-situada se diseña en función de las relaciones a través de diferentes localizaciones entre las que se dan conexiones que definen la razón principal de esta etnografía. En nuestro caso de estudio, la mayor parte del trabajo de campo en India -y su razón de ser- se ha gestado acompañando a personas que, inmigradas a España, estaban temporalmente en la India por negocios, vacaciones o por cuestiones familiares. Esto es consecuencia de los continuos contactos entre los sikhs de Barcelona y la India. Sin embargo, en el caso de Londres, la razón de ser vino por la existencia de conexiones entre las comunidades sikhs británica y barcelonesa, por vínculos de amistad y colaboración existentes, así como matrimonios establecidos entre personas de las dos ciudades. Estas razones y prácticas se enmarcarían en lo que Marcus (1995: 9092) define como "follow the people».

Las técnicas utilizadas durante la investigación también han sido diversas. Si bien la observación participante ha sido fundamental en lugares como Londres, el Panyab y Delhi, la observación directa en Barcelona ha permitido observar y aprehender cómo los individuos actúan e interactúan en espacios variados y tan significativos para la vida tanto familiar como comunitaria y social, como son los templos, sus propios hogares, y diferentes lugares de trabajo. En este sentido, acudir a los gurdwaras cada domingo durante el trabajo de campo en Barcelona ha sido una gran oportunidad e inestimable fuente de información.

Se han realizado también entrevistas semi-dirigidas y abiertas. No obstante, las conversaciones informales siempre han resultado de mayor utilidad al permitir a los y las interlocutoras mostrarse más relajadas y abiertas a hablar con mayor libertad, sobre todo por importantes deficiencias en el manejo del español, el catalán e incluso el inglés, especialmente por parte de las mujeres adultas venidas de la India. En alguna ocasión se ha recurrido a intermediariosquetradujeran del panyabi al castellano o bien al inglés. Asimismo se ha realizado alguna grabación en video. Otro recurso utilizado han sido las consultas en internet, tanto de artículos y publicaciones académicas, como de diarios, páginas webs individuales o de grupos y/o comunidades religiosas autoidentificados como tales, si bien en su consideración y utilización se ha distinguido claramente este último material de las publicaciones con rigor científico. Por último, el diario de campo ha sido una herramienta inestimable en todo el proceso de trabajo. Finalmente, destacar que el diseño metodológico escogido ha sido llevado a cabo por considerarse como el medio más adecuado para conocer a la comunidad objeto de estudio y sus características, a través de los trabajos previos sobre los sikhs en otros lugares (mediante la exhaustiva revisión bibliográfica), y la etnografía multisituada como fuente de información directa de los y las sikhs de Barcelona y sus correlaciones.

A lo largo del trabajo etnográfico se han dado dificultades que cabría mencionar. La primera, venida por la barrera idiomática supuso un hándicap al principio del trabajo para establecer relaciones con mujeres sikhs en Barcelona. Si a este nivel no se daban problemas de comunicación con los varones (que generalmente hablaban inglés o rudimentarios castellano o catalán), ni con los y las sikhs más jóvenes (que hablan tanto el castellano como el catalán casi a la perfección), en el caso de las mujeres sikhs venidas de adultas, comunicarse resultaba muy difícil pues 
generalmente sólo dominan el panyabi, o como mucho también el urdu o el hindi.

Otras circunstancias que también serían negativas se positivizaron aprovechando las coyunturas y las oportunidades al alcance. Como Marcus señala, este tipo de etnografía genera no pocos cuestionamientos o ansiedades (1995: 82-83). El cambio de localizaciones así como la premura que la temporalidad requiere generan sentimientos a veces encontrados o de ambivalencia ya que es condición necesaria de la etnografía multisituada la renegociación constante del rol del/de la investigador/a en función del lugar y el contexto en el que se encuentra (Marcus 1995). Así, si el trabajo en Barcelona requería una importante actitud activa e impulsora, en la India, especialmente por la estructura patriarcal del grupo de estudio, me veía obligada a tener una actitud pasiva e incluso sumisa por mi condición de mujer y a dejarme llevar y a acatar las decisiones que mis anfitriones -varones- tomaban con respecto al qué hacer o dónde, obviando mis intentos de participación o agencia. Así, la mayor complicación del trabajo de campo ha venido sin duda por mi condición de mujer. La sociedad panyabi en general está inmersa en una estricta estructura patriarcal en la que el papel de la mujer queda generalmente relegado no solamente a un segundo plano, sino que sus opiniones, decisiones o voluntades son apenas tenidas en cuenta u obviadas de forma sistemática. Si bien mi condición de mujer me ha permitido acercarme, relacionarme, interaccionar y trabajar con gran facilidad con las mujeres, esta misma condición imposibilitaba mi acercamiento al mundo de los hombres, que son quienes juegan los roles más activos y ostentan el poder de la toma de decisiones con respecto a la comunidad, la familia, ellos mismos y otros individuos bajo su cargo o responsabilidad. Generalmente, los varones se posicionaban desde una doble actitud hacia mi: o bien me trataban con gran paternalismo y a veces desde posiciones dogmáticas, o bien me ninguneaban, no prestando atención a mis sugerencias, comentarios, preguntas o requerimientos. Esto pudo solventarse gracias a la intervención activa del que por entonces era mi compañero en la vida personal, quien me acompañó durante parte de mi trabajo de campo. Sería por su presencia e intervención que mis preguntas (por él planteadas) en las conversaciones informales que teníamos con los varones sikhs obtenían respuestas, y que mi presencia en el mundo de los hombres tenía un lugar para mí y un sentido para ellos (en tanto que yo figuraba como mujer de y era tratada en tanto que tal). Esto no impedía, no obstante, que continuamente varones sikhs trataran de persuadirme de lo que debía hacer, dejándome claro lo que se me negaba y convenciéndome de lo que de mí se esperaba en tanto que mujer que se relaciona y vive entre los sikhs, esto es, que me comportara como si fuese una más entre ellos.

\section{CONTEXTUALIZACIÓN Y FUNDAMENTACIÓN DEL TRABAJO}

Podemos definir a los sikhs como aquellos individuos que se caracterizan por su pertenencia a una religión determinada (el sikhismo), un origen común (el Panyab ${ }^{7}$ ) y una lengua compartida (el panyabi) a los que además de una cultura compartida une un sentimiento de hermandad que se expande a nivel transnacional y global, en directa relación con unas importantes prácticas migratorias y diaspóricas (Santos-Fraile 2016). Podrían definirse, así, como una comunidad imaginada (Anderson 2000) ${ }^{8}$ a escala global, saturada de relaciones político-identitarias vinculadas, en muchos casos, a diferentes procesos de definición étnica y religiosa, pero también a procesos de reconocimiento y búsqueda de la independencia en la India y la consiguiente configuración de un estado sikh soberano.

La población mundial sikh se estima entre 22 y 25 millones de personas (Jacobsen y Myrvold 2011), de las que 1,5 millones o más residirían fuera de la India y dispersas por el mundo. De ellos, se estima que medio millón de sikhs se encuentran establecidos en Europa, donde las mayores y más antiguas comunidades se encontrarían en Gran Bretaña; pero con un importante y creciente número de comunidades en Europa continental (Cole 2004; Jacobsen y Myrvold 2014 y 2011).

7 Estado del Noroeste de la India que al oeste es zona fronteriza con Pakistán.

8 Ese sentimiento de hermandad que nos lleva pensar en la comunidad sikh global como una comunidad imaginada está en directa relación con el hecho de que ser un o una sikh va más allá de una simple definición de los individuos en base a sus creencias. Un o una sikh se definen también por determinada filosofía y modos de vida que los y las caracterizan. Como señalaba Nikky-Guninder Kaur Singh (2004: 8-9), en los fundamentos de esta ética los pilares sikhs se establecerían en base a la vivencia dentro de su fe, donde la hermandad, la comunidad y la familia se hallarían en el centro del sikhismo, creando así la consideración de los otros y otras sikhs como hermanos y hermanas. 
Si bien tradicionalmente los sikhs habían emigrado a lugares vinculados con su historia como miembros de la colonia británica, las limitaciones en las políticas de frontera de sus destinos tradicionales -Reino Unido, Estados Unidos y Canadá- han hecho que en las últimas décadas se asienten en diferentes países de Europa estableciéndose como minoría religiosa (Jacobsen y Myrvold 2014 y 2011) y donde en muchos casos pasan desapercibidos o bien son confundidos con hinduistas o con seguidores de Bin Landen, viviendo situaciones de racismo e islamofobia extensiva, especialmente desde el 9/11, momento a partir del que la identificación étnica primaría sobre cualquier otro rasgo de la subjetividad (Puar 2018).

Su reciente establecimiento en estos nuevos lugares de destino ha motivado que haya surgido un incipiente pero significativo interés por parte de investigadores y académicos que tratan de estudiar y visibilizar a los sikhs en distintos países europeos (Irudaya Rajan, Varghese y Nanda 2015; Jacobsen y Myrvold 2014 y 2011; Santos-Fraile 2007 y 2016). Aun así, hay países donde a pesar de haber un significativo y creciente número de sikhs aún no hay trabajos que desde la academia o desde investigaciones científicas independientes den cuenta de forma pormenorizada del establecimiento de comunidades sikhs así como de las características particulares con que se asientan en estos nuevos países y de los vínculos y relaciones que mantienen con otros sikhs a nivel transnacional. Tal sería, por ejemplo, el caso de Portugal, donde trabajos como los de Bastos (2010) y Correia y Bastos (2006) muestran la existencia de una comunidad sikh significativa dentro de la diversidad religiosa portuguesa pero sobre la que no existen trabajos específicos. Como también sucede en el caso español -y seguramente en otros países del ámbito comunitario-, su visibilización, conocimiento en profundidad y análisis resultaría de gran interés tanto para el conocimiento antropológico y social así como para las políticas de gestión de la diversidad y la pluralidad religiosa, tanto nacionales como europeas; pues lo que se desconoce no se puede gestionar con eficacia y diligencia, y de ello depende en gran medida lograr una sociedad integradora y plural en su riqueza y diversidad.

\section{CONTEXTUALización DE LA COMUNIDAD SIKH DE BAR- CELONA}

La comunidad sikh de Barcelona pertenece a un colectivo mucho mayor que utilizando los términos de
Fredrik Barth (1976) podemos definir como grupo étnico discreto con una importante trayectoria diaspórica, esto es, la comunidad sikh global (Jacobsen y Myrvold 2014 y 2011). Asimismo, encontramos que la forma en que la comunidad sikh se establece en Barcelona tiene ciertas analogías con los modos en que los sikhs se han asentado en otros lugares de Europa $^{9}$ y otras comunidades religiosas del sur de Asia se han establecido en otras ubicaciones (como Estados Unidos, Reino Unido o Canadá) como muestran los trabajos de Judith Brown (2006) para comunidades del sur de Asia, Ballard y Ballard (1979) para los sikhs en Leeds y Gerd Baumann (2003) para Southall, entre otros. Sin embargo, también se dan notables diferencias con lo expuesto en estos trabajos. Como Shinder S. Thandi (2014) sugiere, no se pueden hacer generalizaciones sobre los sikhs en Europa, ya que se encuentran situaciones muy divergentes dadas las diferentes fases de desarrollo en que se encuentran en el proceso migratorio, son diversas las razones para su establecimiento en los distintos lugares y están expuestos a diferentes situaciones de «legalidad» en los diferentes países. Tras la investigación realizada podemos hablar, por tanto, de unas características propias y distintivas de la comunidad sikh de Barcelona, destacando de forma significativa el relevante papel que aquí han tenido y tienen la creación de los gurdwaras, así como las distintas negociaciones que en tanto que individuos y comunidad llevan a cabo. Estas negociaciones van desde las relaciones estratégicas con miembros de la población local que se acercaron a una especie de "sikhismo occidentalizado ${ }^{10}$ y que podemos entender como prácticas o procesos de hibridación (García Canclini 2005) o como puntos de encuentro o intersticios (Benach 2005).

\section{COMUNIDADES GLOBALES: PROCESOS MIGRATORIOS Y DIÁSPORA}

Pierre Bourdieu (1991 en Sayad 2006), en el prefacio del libro de Abdelmalek Sayad, hace notar que en las ciencias sociales existen muchos trabajos sobre el fenómeno migratorio que lo abordan desde las

9 Ver Jacobsen y Myrvold 2014 y 2011.

10 Siguiendo a Edward Said (2002), entendemos aquí «occidente" y lo «occidental» como un espacio o campo de valores y representaciones (que se correspondería con determinada delimitación geográfica) y que es construido a partir de la definición histórica, etnocéntrica y por oposición de "oriente" y "el orientalismo" en términos de otredad. 
problemáticas que a éste se asocian, situándose así desde perspectivas etnocéntricas y reduccionistas. Frente ello, Sayad propone el abordaje del fenómeno migratorio como un hecho social total (Mauss 1979) y desde una mirada interdisciplinaria. Este trabajo, por la consideración de los diferentes factores que en él se observan (movilidad, religión, etnicidad, interacción y género, entre otros) nos permite mirar al fenómeno migratorio desde una perspectiva holística. Destacamos asimismo la importancia del concepto de movilidad humana transfronteriza dentro de los estudios de migración y consideramos a los sikhs como «mobile people» (McLeod 1997: 251) o "people on the move» (Jacobsen y Myrvold 2011: 1). Pues como bien señala Sayad, el hecho migratorio ni es individual ni tiene que ver exclusivamente con el lugar de acogida o de partida, sino que se inserta en un proceso mucho más amplio que conecta el lugar desde el que se emigra al lugar en el que el inmigrado se establece, y donde se requiere de: a) toda una infraestructura de desplazamientos que precisa itinerarios convenidos de antemano; b) contactos institucionalizados que implican redes basadas en la solidaridad étnica o religiosa, el parentesco - la procedencia local, incluyendo recientemente mafias que se han especializado en la organización de esta infraestructura; y c) una organización informativa en el lugar de llegada -bien autóctona o bien del lugar de origen de los migrantes- para orientar, entre otras cuestiones, sobre eventuales puestos de trabajo, alojamiento y asesoría social y jurídica. Todos ellos elementos que, observados desde una escala transcontinental, permiten entender el fenómeno migratorio como sistema y campo unificado, y no solamente como un cúmulo de iniciativas coyunturales (Provansal 2005). En este sentido, y como el trabajo de campo ha puesto de manifiesto, los sikhs participan de redes que desde el lugar de salida y en connivencia con personas ya asentadas en el lugar de llegada, gestionan los viajes y destinos (al principio vía familiares, otros compatriotas y correligionarios, con visados de turista, pero en los últimos años recurriendo en muchos casos a mafias ${ }^{11}$ como última alternativa posible). Asimismo disponen de los gurdwaras como lugares en los que la información y los contactos fluyen materializándose en lugares donde asesorarse, comer, dormir, trabajar y llevar a cabo las gestiones necesarias para el establecimiento

11 Entendemos aquí por "mafias» a redes institucionalizadas con infraestructuras organizadas que con fines lucrativos se dedican al tráfico de personas mediante el traspaso ilegal de las fronteras. y asentamiento en el lugar de llegada. Pues como ya afirmara Sayad (2006: 17), tanto la lengua (vinculada en nuestro caso a la idea de origen común) como la religión (y sus instituciones), se convierten en espacios de relaciones múltiples por los que los migrantes transitan y de los que se sirven en sus movilidades, tanto físicas como sociales. $Y$ entendemos aquí la religión como un fenómeno histórico y transcultural, íntimamente vinculado a la vida social y al poder (Asad 1993) que, como la define Mónica Cornejo Valle,

\begin{abstract}
«Es una realidad polisémica que afecta a cualesquiera de las combinaciones posibles entre creencias en lo sobrenatural, actitudes rituales, emociones ante la trascendencia y las instituciones que en cada sociedad han ido asumiendo la regulación (y la propia definición folk) de los fenómenos anteriores. Algunos de estos fenómenos son colectivos y otros individuales, unos excepcionales y otros cotidianos, unos son psicológicos y otros plenamente materiales, pero los hechos y representaciones que consideramos religiosos, en conjunto, no comparten un único rasgo universal en este sentido (no son solo colectivos o solo inmateriales, etc.)». (Cornejo Valle 2016: 86).
\end{abstract}

La religión o marco religioso ofrece así un espacio en el que los migrantes e inmigrados creyentes comparten un campo no sólo semántico, sino también de acción, del que se sirven y a partir -o a través- del que se sitúan en los diferentes contextos, también los propios del proceso migratorio. Es en esta línea que nos interesa considerar los planteamientos de Steven Vertovec cuando afirma que lo religioso, del mismo modo que sucede con otras dinámicas socio-culturales, se desarrolla de forma distintiva en los ámbitos de la migración y los estatus de minoría, las diásporas y el transnacionalismo (Vertovec 2009: 136); fenómenos que además se solapan y sobre los que las transformaciones religiosas tienen lugar (Vertovec 2009: 147).

Todas ellas categorías que son de fundamental importancia para conocer sobre nuestro grupo objeto de estudio, pues, aunque a distintos niveles, refieren al marco contextual de prácticas y relaciones en el que los sikhs que habitan Barcelona se ubican y desarrollan.

\section{LA COMUNIDAD SIKH: UNA COMUNIDAD RELIGIOSA, TRANSNACIONAL Y DIASPÓRICA.}

El concepto de comunidad puede resultar polisémico al significar realidades diferentes en cuanto a las características y a la vinculación existente entre el conjunto de personas al que refiere. Para 
Godelier (2010: 6-7), por ejemplo, una comunidad es un grupo que comparte tradiciones, maneras de vivir, etc, (una cultura, en definitiva) y que coexiste en un espacio definido con otras comunidades en el seno de diferentes sociedades y Estados. En nuestro caso de estudio, en cambio, los límites de la comunidad no se definen por el lugar donde sus miembros están asentados o habitan, sino por la religión y el origen, incluyendo, así, a cualquier persona, en cualquier lugar del mundo, que se identifique con esto $^{12}$ (Santos-Fraile 2007). Esta conceptualización de comunidad, estaría asimismo en consonancia con la definición que Judith Brown establece para las comunidades del sur de Asia en general, y que las aleja de la concepción como minorías étnicas comúnmente utilizada. Brown pone el énfasis en su involucración en una densa red de conexiones tanto locales como globales que les convierte en verdaderos transnacionales que responden a oportunidades y desafíos en ambos niveles -el local y el global- y en que son muy conscientes del papel emergente del sur de Asia en un entorno mundial cambiante (2006: 8) ${ }^{13}$.

Al mismo tiempo, la definición que aquí se propone de comunidad es en cierto sentido análoga a la ofrecida por Gerd Baumann cuando refiere a "'selfevident' communities of culture» (Baumann 2003: 72-73), dado que los sikhs se definen a sí mismos y son definidos por otros grupos asiáticos como una comunidad religiosa caracterizada por una cultura propia y distintiva. En otras palabras, esta comunidad se define principalmente en función de criterios religiosos. No obstante, podemos considerar al colectivo sikh que habita Barcelona como perteneciente a una comunidad históricamente migrante y diaspórica mucho más extensa, que tendría un estatus de minoría, pero que pertenece a una comunidad mucho más amplia que se caracteriza por su globalidad y transnacionalidad (Jacobsen y Myrvold 2014). Asimismo, concordamos con Barth (1976) cuando expone cómo la identidad étnica se relativiza en función del contexto como resultado de interacciones que terminan desembocando en

12 Pese a que podemos encontrar un creciente número de personas occidentales, conocidos como gora-sikhs, que se autodefinen como seguidores del sikhismo, no se les incluye dentro de la definición de comunidad sikh puesto que los sikhs de India y los sikhs occidentales no comparten ese sentimiento mencionado de identificación y pertenencia a una misma comunidad. oposiciones, lo que nos lleva a hablar por tanto de identidades flexibles y contextuales, algo que también Jacobsen y Myrvold (2011) muestran.

Si bien para el caso de Southall Baumann (2003) señala cómo el reconocimiento de la comunidad sikh como un "grupo internamente homogéneo, claramente delimitado" viene como parte de un discurso dominante que más tarde se consagraría en la legislación británica, en Barcelona, en cambio, estas características de grupo homogéneo y delimitado se van creando y negociando en las prácticas cotidianas de los miembros de la comunidad. Practicas que, a su vez, serán las mismas a partir de las cuales esta comunidad religiosa negocia su establecimiento en tanto que tal y que son indisociables de una identidad común en base al origen y la fe que cohesiona y genera acciones de vinculación y solidaridad -aunque también limitaciones y obligaciones- en las que los gurdwaras actúan muchas veces como centros neurálgicos.

\section{LOS SIKHS EN BARCELONA}

Según fuentes oficiales del Estado español, en Barcelona habitan en la actualidad más de 18.700 personas de origen hindú (que podemos considerar, en su gran mayoría, hinduistas y sikhs), más de 26.300 en Cataluña, y de 49.100 en todo el Estado ${ }^{14}$. Si atendemos, en cambio, a los datos ofrecidos por el Institut d'Estadistica de Catalunya y su evolución, la cifra de población extranjera de origen hindú para el año 2000 (primera fecha con datos disponibles) era de 799 hombres y 529 mujeres, sumando un total de 1.328 personas, lo que suponía el $0,73 \%$ sobre el total de extranjeros en Cataluña. Ya para el año 2019, las cifras que señalan son 17.222 hombres y 9.139 mujeres, sumando un total de 26.361 personas de origen hindú que supondrían el 2,27\% sobre el total de extranjeros en la región. Centrándonos en la provincia de Barcelona, las cifras para el año 2000 señalan 542 hombres y 385 mujeres, que sumarían un total de 927 hindúes en la provincia, lo que para entonces supondría el $0,70 \%$ de la población extranjera en Barcelona. Ya para el año 2019, los datos registran la existencia de 12.485 hombres y

14 Datos obtenidos del Padrón Continuo de 2019, Instituto Nacional de Estadística (INE). Disponible en: <http:// www.ine.es>. Visitado el 16/07/2020. 
6.248 mujeres, sumando un total de 18.733 hindúes y que supondrían un $2,33 \%$ de la población extranjera en la provincia ${ }^{15}$. Si bien estas cifras corresponderían a los datos que se tienen de personas venidas de la India, con independencia de su adscripción religiosa, las estimaciones hechas desde el Consell Sikh de Catalunya ${ }^{16}$ y otras instituciones en 2007 situaban ya de forma específica el número de sikhs -considerando tanto a «regulares» como «irregulares»- en 5.000 para Cataluña y en 10.000 para toda España ${ }^{17}$.

Así, a pesar de su invisibilidad, el número de sikhs que habitan en Barcelona y en sus alrededores ha ido creciendo notablemente en las últimas décadas, con un notorio incremento de mujeres debido a la reunificación familiar, a lo que se une el crecimiento de una aún muy tenue segunda generación que se abre camino como habitantes que desarrollan una capacidad dual: la de desenvolverse con soltura en el que fue un nuevo y diferente contexto para sus padres, y el aprendizaje, manejo y reproducción de la cultura parental con la que se identifican y que valoran como lugar en el que se construyen significados.

El número de gurdwaras también ha ido aumentando de forma significativa en los últimos años. El trabajo de campo realizado para esta investigación ha desvelado que en la actualidad se encuentran -al menos- 22 gurdwaras repartidos por la geografía española ${ }^{18}$, si bien la mayor concentración

15 Fuente: Idescat, Instituto d'Estadistica de Catalunya, Generalitat de Catalunya. Disponible en: <http:// www.idescat.cat $/$ poblacioestrangera $/$ ?nac $=d 410 \&-$ $b=1 \& t=2000 \& x=7 \& y=6>$. Fecha última consulta: $16 / 07 / 2020$.

16 Órgano de representación creado en 2005 para actuar como interlocutor entre los Sikhs y las administraciones.

17 Esta es una estimación hecha por algunas instituciones para sus cálculos propios. Al no haber datos estadísticos que discriminen a la población por creencias religiosas o por lugar de nacimiento en la India es imposible obtener datos más precisos. A esto se une que muchos sikhs se encuentran en situación «irregular», siendo también invisibles en las estadísticas oficiales.

$18 \mathrm{Si}$ bien es difícil conocer con exactitud el número de gurdwaras que actualmente existen en el país por la falta de trabajos que los cuantifiquen, el sabemos de la existencia de 22 templos ubicados en la geografía española, estando localizados en Alicante, Barcelona, Girona, Málaga, Madrid, Palma de Mallorca, Murcia y Valencia. Como decimos, es difícil saber la existencia exacta del número de gurdwaras y la evolución temporal de estos centros si tenemos en cuenta que no todos ellos están registrados como entidades religiosas en el se encuentra en Cataluña. Su creación, además de responder a la creciente población y dispersión sikh y a su cada vez mayor organización en tanto que comunidades locales, también se debe a factores como los conflictos relacionados con el origen de casta, que en el caso de Barcelona han tenido una importante incidencia en la creación de nuevos templos.

\section{EL PROCESO MIGRATORIO HASTA BARCELONA}

Una de las primeras cuestiones a tener en cuenta al considerar de forma específica el proceso de los sikhs venidos a Barcelona, es el cambio que se ha producido en los flujos migratorios de la diáspora sikh. Si bien, como se hacía notar más arriba, existe una tradición migratoria panyabi que, aunque con antecedentes, se remonta sobre todo a la época de la colonización británica, la globalización ha conllevado una importante diversificación de las pautas migratorias a nivel internacional, donde la cantidad de flujos, su volumen, sus características así como los países emisores o receptores han conocido una diversidad sin precedentes que además se ha dado a una velocidad sin igual. Esto, sin lugar a dudas, no ha dejado de afectar a aquellos sikhs que emigraban. En esta misma línea, el importante endurecimiento y restricción en las políticas de inmigración tanto del centro de Europa como de Estados Unidos desde

Ministerio de Justicia, donde sólo constan 13. Algunos gurdwaras, de hecho, optan por no registrarse o hacerlo como centros culturales para evitar los costos de registro. No obstante, según los datos del Ministerio de Justicia, el primer gurdwara registrado fue el de Badalona, siguiéndole el de la calle Hospital (en BarceIona), ambos en 2009, y los demás por este orden: en 2010 uno de Palma de Mallorca, el de Torre Pacheco (Murcia) y el de Valencia; en 2011 el de Santa Coloma de Gramanet (Barcelona) y el de Palma de Mallorca; en 2012 el de Vic (Barcelona), el de Salt (Girona), el de Olot (Girona) y el de Alicante; ya en 2016 se registraría el de L'Hospitalet de Llobregat (Barcelona) y en 2018 el de Torrevieja (Alicante). Sin embargo, si consideramos los datos que ofrece el Observatorio del Pluralismo Religioso en España (http://www.observatorioreligion. es), no es hasta 2012 que aparecen registrados los primeros gurdwaras, concretamente 12: 3 en Girona, 5 en Barcelona, 1 en Valencia, 2 en las Islas Baleares y 1 en Murcia; en 2013 aumentan su número en 16; lo incrementan en 2016 a 20; para 2017 especifican la existencia de 11 templos en Cataluña, 4 en Valencia, 2 en Islas Baleares, 2 en Madrid y 1 en Murcia; ya para 2018 se incrementan en 22 y en 2020 cifran en 23 el número de gurdwaras en el Estado español. 
comienzos de la década de los setenta, han llevado a la aparición de nuevos países de destino como fue el caso del Estado español, con una política de inmigración poco definida hasta prácticamente 1985. Así, cuando las redes importantes de emigración de sikhs desde la India se habían dirigido tradicionalmente a países como Estados Unidos, Canadá y Reino Unido, el cambio en las mencionadas circunstancias les ha hecho redefinir sus lugares de destino. Se dirigieron entonces hacia países en los que, como es el caso del Estado español, en muchos casos se desconocía sobre su religión, tradiciones y formas de pensar y hacer -su cultura, en definitiva- y a los que han debido de adaptarse o integrarse llevando a cabo procesos de mediación y negociación (Santos-Fraile 2016). Tanto es así, que a pesar de que en sus preferencias siguen estando los tres países antes mencionados, en la actualidad cada vez se producen más movimientos hacia Sudamérica y Europa continental (Cole y Sambhi 2006; Jacobsen y Myrvold 2014 y 2011).

\section{LOS SIKHS DESDE LA INDIA HASTA BARCELONA}

Los sikhs que han venido a Barcelona son fundamentalmente migrantes laborales, aunque también se dan casos relacionados con la disidencia política o religiosa, fruto de las consecuencias históricas de la lucha activa por el separatismo; tal fue el caso de los conflictos religiosos a raíz del asesinato de Indira Gandhi por parte de dos sikhs de su guardia personal.

Como ejemplo del primer caso -de disidencia política- estaría Yasín, quien fue activista por la independencia del Panyab y que cuando se supo buscado por la policía eliminando aquellos elementos que lo caracterizaban como varón sikh ${ }^{19}$ huyó del país para establecerse en Suiza. Tras pedir el visado en distintas embajadas, fue la española la única que se lo concedió, así que vino aquí. Viendo que en España «le trataban bien» y que en Suiza no encontró el trabajo prometido, decidió quedarse.

El segundo caso -conflicto religioso- lo ejemplifican Anarjit y su familia, que venían de viaje mezclando negocios y turismo -su padre exportaba productos de artesanía desde la India a países Europeos y entre ellos

19 Nos referimos a las $5 \mathrm{ks}$, elementos religiosos que identifican a los sikhs (el kesh, o pelo sin cortar; la kangha o peine de madera que sujeta el pelo; el kirpan o daga; la kara o pulsera de acero, y la kachera o tipo de calzón muy específico). a España-. Estaban en Barcelona cuando ocurrieron los altercados tras el asesinado de la Primera Ministra Hindú y que llevaron a violentas persecuciones sobre los sikhs. La familia de Anarjit decidió por tanto quedarse en Barcelona hasta que la situación en Delhi volviera a la normalidad y finalmente se han establecido de forma permanente.

Es un hecho generalizado que los sikhs establecidos en el país no eligieron España como lugar final de destino. Vinieron primero como turistas, y más tarde asentados y con estabilidad laboral en el país, sirvieron como atracción para sus compatriotas. Las motivaciones que les traen hasta aquí están directamente relacionadas con la búsqueda de una mejora socio-económica, donde el prestigio -izzat ${ }^{20}$ que implica la migración, así como la adquisición de tierras y/o la construcción de una nueva y ostentosa casa en el lugar de origen son incentivos decisivos (también asociados al prestigio); hecho que por otro lado es común a numerosos colectivos inmigrantes.

Si bien algunos vinieron hasta aquí -y lo siguen haciendo- como forma de acceso a Europa (con la intención de a largo plazo instalarse en alguno de sus destinos de preferencia), otros lo hicieron atraídos por las opciones laborales. Parece una tónica general que cuando estos sikhs se plantean el proceso migratorio, la idea que tienen en mente es la llegada a Europa, a la que parecen considerar como un todo donde las partes (o los países en concreto) son sólo lugares circunstanciales a los que se va o se llega en función de las opciones más estratégicas. Tanto es así que no tienen dificultades para moverse de unos países europeos a otros en función de las oportunidades laborales, o las posibilidades de regularizar su situación. No son pocos los sikhs que actualmente instalados en Barcelona han pasado antes por otros países europeos. Este sería el caso de Aarini, por ejemplo, que proveniente de Banga -en el distrito de Nawan Shahar-, entró legalmente en España en 1990 tras haber estado en Alemania, Bélgica y Francia donde no consiguió «regularizar» su situación. También sería el caso de Davinder, quien después de un tiempo asentado en España y mientras espera el momento de regularizar su situación aquí para solicitar la reagrupación familiar, decidió trasladarse temporalmente y abrir una gasolinera en Bélgica con su hermano debido a la falta de trabajo

20 Izzat puede traducirse como prestigio, honor y estatus. 
por la situación de crisis del país. Estos traslados más o menos temporales especialmente a países como Francia y Alemania son una estrategia frecuente entre muchos varones sikhs a partir la fuerte crisis económica en el Estado Español. Es, de este modo, que muchos varones ya instalados y con intención de permanencia aquí, se desplazan por otros países europeos sin estrategias claras de regreso, sino abiertos a la movilidad siempre en función de las circunstancias que más les favorezcan.

Si al principio venían con visados de turista o de estudios, la siguiente opción fueron los contratos de trabajo que les facilitaban sus propios paisanos ya asentados y que normalmente regentaban algún negocio ${ }^{21}$. A partir del auge constructivo y laboral generado en Barcelona y alrededores de cara a las Olimpiadas del 92 y la reestructuración que se haría en la ciudad, el efecto llamada fue un importante aliciente para venir a la ciudad e instalarse. Los procesos de regularización llevados a cabo por el Estado también fueron un aliciente para aquellos sikhs que de forma «irregular» ya estaban en otros países de Europa. Sin embargo, con el endurecimiento de las políticas de entrada y regularización en el país, la situación de acceso se fue recrudeciendo hasta tal punto que desde hace algunos años la vía de llegada pasa más por el recurso a las mafias ${ }^{22}$ que normalmente les traen hasta Ceuta o Melilla. Esto ha favorecido situaciones de extrema dificultad para muchos sikhs que emprendieron el viaje migratorio con las mafias como pasaporte, y que han tenido que pasar por situaciones tan extremas que según algunos de ellos narran ha llevado a no pocos hasta la muerte. Así recogía en su día el testimonio de Garry, de unos 27 años, que se encontraba en el CETI (Centros de Estancia Temporal de Inmigrantes) de Melilla y explicaba que llevaba cuatro años allí. Se lamentaba de que las autoridades no se hacen cargo de ellos. Explicaba que había pagado $12.000 €$ a las mafias en la India para que le trajeran a Europa, bastante más de lo que le pedían al principio. Les trasladaban en camiones y en coches, a oscuras, y llegó aquí

21 Estos contratos de trabajo en muchos casos derivan en situación de explotación laboral hacia sus propios paisanos, entrando por tanto en la categoría de trata de personas.

22 Según algunos informantes la cantidad que se paga a las mafias ya sea para conseguir un visado, o bien para traerlos hasta aquí oscila entre los 25.000 y los 60.000 euros. cruzando por varios países de África, hasta que les dejaron en Melilla. Estuvo en el Sahara dos meses casi sin comer ni beber y debido al calor tuvieron que quitarse el turbante y cortarse el pelo. Su intención era llegar a Inglaterra, pero ahora prefiere quedarse aquí. Asimismo, explicaba que cuando vienen no lo hacen pensando en llegar a un país en concreto, sino que esto les da igual. Que no pagan por un destino, sino por que les traigan a Europa.

Según sus propios testimonios, estos viajes, en travesías de hasta tres años, suelen llevarles desde sus lugares de origen hasta África, donde, en condiciones infrahumanas y cruzando el desierto del Sahara, pasando por países como Níger, Mauritania y Argelia, llegan hasta Marruecos. Una vez allí consiguen acceder a Ceuta o Melilla, donde los dejan haciéndoles creer que están en tierras continentales europeas. Como fue el caso de Garry, en muchos casos terminan en los CETIS desde donde serán deportados a su país de origen.

\section{LLEGADA Y ASENTAMIENTO EN BARCELONA}

"Sunjeet ${ }^{23}$ me explicaba que vienen a España porque por la situación legal no te ponen muchas trabas, y que lo de los papeles se puede arreglar. Utilizan España como entrada para el arraigo (lo consiguen viviendo 3 años aquí, con la tarjeta sanitara y empadronándose vía la Cruz Roja, sin necesitar un domicilio fijo). Así, se consigue el arraigo con el pasaporte, con un contrato de trabajo, con el certificado de antecedentes penales y con la tarjeta sanitaria. Dice que en el CAP se observa cómo en los últimos años ha habido muchísimos registros de gente que realmente no asiste a consultas, sino que se registran para dejar constancia y años más tarde vuelven para acreditar que están aquí, pero en ese tiempo no están aquí, sino que van a otros países. Cuando hemos hablado del tema de las mafias, dice que son los propios indios y pakistaníes los que trafican con sus paisanos». [Notas de Campo, Barcelona, Diciembre de 2009].

Este extracto evidencia cómo más que el lugar de destino, es la estrategia de asentamiento lo que prima a la hora de establecerse en el país. Asimismo, es interesante señalar con respecto a los sikhs

23 Sunjeet es una colaboradora principal, cuyo conocimiento de la población inmigrada asiática se pone en valor especialmente por desarrollar labores de intérprete y traductora y por trabajar en un Centro de Atención Primaria (CAP) en el centro de Barcelona. 
asentados Barcelona y su periferia que, a diferencia de lo que Roger Ballard y Catherine Ballard (1979: 21-56) encontraron entre los sikhs en Leeds, aquellos que vienen a Barcelona una vez aquí no se lo plantean como una estancia temporal para ahorrar dinero y volver a casa, sino que tratan de establecerse y regularizar su situación cuanto antes para traer a sus familias nucleares $y$, de ser posible, también parte de su familia extensa ${ }^{24}$. Tras esta práctica está la consideración de Barcelona en particular, y Europa en general, como una tierra de oportunidades para ellos y sus familias. Aunque esto no implica que a veces el establecimiento y la regularización de su situación en España en particular deje de plantearse como una estrategia para luego acceder a aquellos terceros países que son considerados lugares que otorgan un plus de prestigio. Lo que sí resulta significativo destacar es que los planteamientos de los sikhs que habitan Barcelona contradicen también el postulado de Sayad (2006). Si Sayad incide en que el estado de aquel que migra es siempre provisorio, su razón de ser sería el trabajo, y en él subyace la idea perenne de retorno, en nuestro caso de estudio esta idea de retorno no parece estar en la mente de los sikhs. El regreso definitivo a la India sería visto como un fracaso del proyecto migratorio.

\section{CARACTERÍSTICAS ESPECÍFICAS DE LA COMUNIDAD EN BARCELONA}

La comunidad sikh en Barcelona se caracteriza por ser principalmente población masculina en edad laboral, en una proporción que podemos estimar de un $70 \%$ de varones por un $30 \%$ de mujeres ${ }^{25}$. Sus procesos migratorios son predominantemente masculinos y las mujeres vienen años más tarde mediante la reunificación familiar. La dinámica migratoria familiar consiste en que primero emigra el

24 Si bien es cierto que hay un número importante de varones que no han traído a su familia no podemos afirmar que esto no sea cuestión de tiempo. Habrá que ver qué sucede y cuáles son las estrategias que se desarrollan al respecto en el caso concreto de esos varones que de momento no parecen apostar por el reagrupamiento familiar.

25 Dado que no existen datos que puedan mostrar esta información, esta estimación estaría basada en las propias observaciones, en conversaciones con los miembros de la comunidad y en la entrevista a Agusti Iglesias, técnico en Políticas Migratorias de la Direcció General d'Afers Religiosos de la Generalitat de Catalunya. varón y una vez regularizada su situación, aquellos que tienen esposas e hijos los traen; y aquellos que son solteros van a la India, donde contraen matrimonio previo concierto por los parientes con alguna mujer de allí, y después de algún tiempo se traen a su nueva esposa ${ }^{26}$. Los sucesivos procesos de regularización y de reunificación familiar han facilitado que se haya ido produciendo un importante crecimiento en la proporción de mujeres sikhs inmigradas cuyo número ha ido variando de forma proporcional al número de varones inmigrados, a su mayor estabilidad laboral y su acceso al matrimonio; un fenómeno que nos permite hablar de una lenta pero progresiva feminización de la inmigración panyabi en nuestro país.

Otra de las características esenciales a señalar es que, aunque los sikhs comparten un mismo origen geográfico, la misma religión -con matices y divisiones internas- y la misma cultura, existe una importante diversidad dentro de la comunidad. No sólo por el lugar específico de origen (existen sustanciales diferencias entre los sikhs de las zonas rurales y los de las urbes más importantes del Panyab y Delhi, por ejemplo), sino también por su casta de pertenencia o su bagaje socio-económico, hechos que marcan distinciones y posiciones fundamentales en el seno de la comunidad. Pese al hecho de que los sikhs gozan y han gozado históricamente de un importante reconocimiento como buenos y prósperos trabajadores, podemos encontrar importantes diferencias entre aquellos que poseen mayores recursos económicos (lo que les permite viajar a menudo y tener un buen nivel de vida), y aquellos que viven en situaciones mucho más precarias (normalmente son los recién llegados) y que podemos observar, por ejemplo, vendiendo latas de cerveza por las calles y bocas de metro más céntricas y concurridas de Barcelona. Los propios sikhs asumen que existe además una diferenciación fundamental que condiciona sobremanera su situación y sus potencialidades, y es la de «tener o no tener papeles»: si su situación está regularizada, podrán optar a trabajos en mejores condiciones, podrán tener acceso al alquiler de una vivienda, podrán, en sus propias palabras "comer bien y estar felices», como

26 Como se analiza con detalle en el capítulo cinco de mi tesis doctoral (2016), también se dan casos de varones sikhs que contraen matrimonio temporalmente con mujeres europeas con objeto de regularizar su situación, dando lugar, en algún caso, a situaciones muy particulares. 
decía Harjinder. Estar en una situación «irregular» en este sentido, impide el acceso a una vivienda, a un trabajo en mejores condiciones de estabilidad y salario, y garantizar el sustento y, por extensión, una calidad de vida digna, tanto para ellos como para sus familias en la India. A ello se une, además, la frustración generalizada por no satisfacer nunca suficientemente las expectativas de los familiares que allí se quedaron. Un lamento general, tanto entre aquellos que han conseguido estabilidad económica y laboral aquí, como entre aquellos que no, viene por la incomprensión por parte de los familiares (y también de amigos) en la India porque no envían suficiente dinero. Esta situación de frustración y desencuentro ${ }^{27}$ es generalizada entre los sikhs que están aquí, con independencia de su situación y solvencia económica y de la cantidad de dinero que regularmente envían a la India.

En cualquier caso, podemos encontrar una amplio abanico entre los sikhs de Barcelona, que va desde empresarios con diversificados negocios, lo que les otorga una más que solvente posición económica y que emplean a sus familiares y compatriotas en hostelería, agencias de viaje, redes de comercio, la construcción o la confección de textiles tradicionales hindúes (a los que a su vez en muchas ocasiones alquilan habitaciones, obteniendo así beneficios extra), hasta trabajadores asalariados en la construcción, en fábricas o en el sector servicios, incluidos trabajadores en condiciones de importante precariedad o desempleados. También existe una gran diversidad en los niveles de formación cualificada. A pesar de que no todos ellos tienen estudios superiores, sí hay un número importante, sobre todo de jóvenes, cualificados profesionalmente y/o con estudios medios en situación irregular que desempeñan trabajos muy precarios.

En cuanto a las mujeres se refiere, las de mayor edad y que vinieron ya adultas de la India, habitualmente se dedican a las tareas domésticas o como modistas de sus paisanas; pero igualmente podemos hablar de una importante diversidad. En los casos de las mujeres que llevan largo tiempo viviendo aquí es más fácil encontrarlas trabajando en pequeños

27 Tanto desde Barcelona, oyendo conversaciones telefónicas, como en la India, siendo testigo directo, he podido presenciar desencuentros y discusiones familiares a cuenta de la cantidad de dinero que se espera y se demanda que envíen a la India. establecimientos familiares o en el servicio doméstico por cuenta ajena. Aquellas que trabajan de cara al público destacan por un buen dominio del lenguaje local y nacional, hecho que no se da entre las que se dedican a las tareas del hogar.

Una característica común que sí encontramos entre las mujeres es su formación (entre las más jóvenes, universitaria, y, formación media o profesional entre las más mayores) y la adquisición de una profesión previas al matrimonio que difícilmente desarrollarán.

Si bien en Barcelona la familia trata de reproducir y mantener los roles tradicionalmente asignados a hombres y mujeres, la comunidad, -como si de una extensión de la familia se tratara- adquiere un rol esencial y se encarga de controlar y velar por el cumplimiento de estos roles. Es aquí donde cobra sentido la sangat o congregación, que es el lugar social en el que se toman las decisiones concernientes a la comunidad, y que es también el espacio para la solidaridad, para la transmisión y el mantenimiento de las tradiciones. Todo ello en gran medida se materializa en los gurdwaras como espacios físicos.

Si bien en Barcelona la comunidad es elemento fundamental de articulación y control de sus miembros, en su seno se dan divisiones internas que se tratan de mantener e incluso potenciar precisamente para estructurar internamente al grupo. Refiero concretamente aquí a la división de la comunidad por castas lo que está en relación con la creación de gurdwaras, ya que al gurdwara al que se asiste se determina en gran medida por relaciones de afinidad o parentesco, lo que en última instancia remite a vínculos de casta.

En la actualidad hay 7 gurdwaras en la provincia de Barcelona. El primer templo, en Barcelona ciudad, el Gurdwara Nanaksar se creó en 1992 en el Barrio de Sants y se ubicaba en la calle Sagunto, 75. Luego vendría el que se encuentra en el barrio del Raval, el Gurudwara Gurdarhshan Sahib, en la calle Hospital 97 y más tarde, espaciados en el tiempo se crearían dos en Badalona, el primero el Gurudwara Gursangat Sahib en la calle Ramón Llull, 5 y el segundo, con el nombre de Gurdwara Sri Guru Ravi Das Bhavan en la calle Juan Sebastián Bach, 8. Durante bastante tiempo se estuvo gestionando la apertura de otro templo más en Badalona, estimulado por el importante crecimiento y concentración de sikhs que allí se da, puesto que eligen con bastante frecuencia este municipio cuando poseen los recursos suficientes para adquirir una vivienda en 
propiedad. Finalmente se habilitarían dos templos más en una localidad cercana, en Santa Coloma de Gramanet, el Dhan Shiri Guru Granth Sahib, en Calle Firal 29 y el Gurudwara Guru Ladho Re en Parc de Can Zam s/n. También se trabajó para habilitar un espacio en Hospitalet de Llobregat, a donde por cuestiones de espacio se trasladaría el templo de Sants y donde se da la mayor concentración de extranjeros provenientes de la India en el extrarradio barcelonés. Tras la adquisición de un local que tuvo un costo de más de 420.000€, durante largo tiempo tuvieron que desistir en el intento al no conseguir las licencias necesarias por parte del consistorio, lo que conllevaría importantes desavenencias y conflictos dentro del grupo asiduo a este templo, que terminaría dividiéndose. Finalmente se abriría en el Carrer Rafael Campalans 26. También se abriría el templo Gurudwara Guru Teg Bahadur SahibVic en la calle de Rafael Gay de Montellà 6, en Vic ${ }^{28}$.

Aparte de los templos ya existentes, entre las aspiraciones de los sikhs se encuentra la creación de un gran gurdwara en la ciudad -de unos 3.000 m2para lo que tratan de negociar con las instituciones catalanas, solicitándoles un terreno de esa extensión, y argumentando que la comunidad dispone de los recursos necesarios para su construcción para lo que cuentan con apoyo de los sikhs del Reino Unido, lo que refleja los vínculos transnacionales de la comunidad sikh de Barcelona.

En cuanto a la construcción identitaria se refiere, los sikhs de Barcelona se reconocen y auto-identifican como comunidad y en oposición sus otros, que se reifican en el islam como religión y en los pakistaníes (y en algún caso en los marroquíes) como grupo; esto se manifiesta recurrentemente en sus discursos. $\mathrm{Si}$ bien en un principio los pakistaníes fueron aliados o apoyo para el acomodamiento e inserción en la sociedad de acogida, con el tiempo tratan de marcar distancia y distinción. Así, refieren a los musulmanes en general como extremistas religiosos, con grandes

28 A nivel administrativo, y por recomendación de la Direcció General d'Afers Religiosos, los gurdwaras se inscriben como asociaciones en el Registro de Entidades Religiosas del Ministerio de Justicia, o se registran como asociaciones culturales en el Registro de Asociaciones del Departamento de Justicia de la Generalitat. Esta segunda opción, que supone una situación de cierta a-legalidad es la normalmente utilizada en Barcelona, al resultar más accesible y menos costosa, además de permitir el acceso a ciertas ayudas y subvenciones. dosis de machismo y muy posesivos con sus mujeres. También, según los sikhs, tanto pakistaníes como marroquíes son racistas para con ellos; así como seres conflictivos con tendencia al robo y las prácticas ilícitas e ilegales.

\section{LA COMUNIDAD SIKH DE BARCELONA EN EL CONTEXTO MUNDIAL}

La vinculación transnacional de la comunidad sikh de Barcelona se pone continuamente de manifiesto desde las relaciones y la solidaridad existentes en la comunidad, que trascienden los límites de lo local. Los sikhs mantienen frecuentes contactos con la familia extensa y el lugar de origen, no solamente a través del teléfono e Internet, sino también enviando remesas regulares que ayudan a mantener, incrementar o mejorar las propiedades y la estructura familiar, pero también para la creación o mejora de otros templos y espacios, como pueden ser colegios y otros centros de formación sikhs, residencias de ancianos o centros sanitarios.

Así, desde Barcelona, se mantienen frecuentes contactos con la comunidad a nivel internacional con la que se colabora económica y éticamente, y de la que se está continuamente informado, ya sea a través de las versiones on-line de los periódicos panyabíes, los programas internacionales que visionan a través de las antenas parabólicas y los contactos frecuentes con los familiares y amigos que permanecen en la India como con aquellos otros miembros que emigraron a otros lugares. Frecuentes visitas a la tierra natal así como a otras comunidades de la diáspora es otro recurso habitual para mantener fuertes vínculos transnacionales.

Especialmente cuando importantes eventos acontecen, como fue el gran langar ${ }^{29}$ que se preparó con ocasión del Forum Universal de las Culturas en 2004 en Barcelona, organizado por sikhs venidos de Birmingham y Canadá debido a la aún precaria organización de la comunidad Barcelona. Un ejemplo más reciente fue el incidente producido el 24 de Mayo de 2009 en Viena cuando algunos sikhs ortodoxos dispararon a dos líderes espirituales de la comunidad ravidassia $^{30}$ considerados como herejes por los

29 Comida comunitaria.

30 Sub-grupo religioso que se caracterizada por ser seguidores de Ravidass, considerado por éstos como gurú (maestro dotado de una importante sacralidad), pero por los sikhs más 
primeros. Como consecuencia de este hecho, muchos seguidores se movilizaron a nivel internacional y la violencia se desató en el Panyab, donde el gobierno tuvo que declarar el toque de queda y enviar al ejército para restaurar el orden.

Asimismo, existe una especie de organización virtual y supranacional que de forma constante promueve y gestiona información manteniendo a los sikhs de los distintos países en relación, y donde la comunidad sikh de Barcelona participa activamente. Se utiliza para ello internet ${ }^{31}$ como un recurso fundamental, pero no único. Existe un registro oficial con sede en el Panyab con información y contacto de todos los gurdwaras en el mundo. En este se guarda una relación de cada templo que se abre, de forma que mantienen y ofrecen información constante sobre el número de gurdwaras fuera de la India ${ }^{32}$ y su ubicación, y donde aparecen registrados algunos de los templos de la ciudad condal. La utilización de antenas parabólicas es un recurso informativo muy utilizado al permitir la visión de programas realizados en distintos países y que luego serán retransmitidos por toda una serie de canales panyabíes. Así como en Barcelona en distintas ocasiones han venido reporteros desde Gran Bretaña para grabar en los templos o durante alguna celebración, también hay corresponsales en Barcelona que se encargan de publicar $y$ transmitir para medios informativos sikhs a nivel global. También la radio ayuda a que fluya la información gracias a programas producidos por los mismos panyabíes, tanto a nivel local como internacional.

\section{CONCLUSIONES FINALES}

Así como sucede en el propio Panyab, existe una gran diversidad entre los sikhs de la diáspora en

ortodoxos como bhagat, es decir, líder espiritual, pero no gurú. Este grupo se caracteriza además por ser principalmente de casta baja, como lo fue el propio Ravidass.

31 Así, se pueden encontrar artículos publicados en webs internacionales donde se solicita ayuda para cubrir las necesidades de sikhs en la diáspora, como sucedió en Valencia, donde tenían serias dificultades económicas para cubrir las necesidades del gurdwara. En este caso, por ejemplo, aportaron dinero sikhs desde lugares tan alejados como Corea del Sur o comunidades como la de Coventry.

32 Este registro permite asimismo el control financiero de los templos desde el Panyab, así como la obtención de una parte de los ingresos que se demandan desde el centro de gobierno de los sikhs. función del lugar social que ocupan, la casta y la opción o las condiciones religiosas, lo que no impide que mantengan fuertes vínculos con sus parientes y lugares de origen (Pániker 2007: 321-326; Singh Tatla 2004: 63-111). Por otro lado, las nuevas tecnologías, la mejora en las comunicaciones y la popularización y mayor acceso a los medios de transporte no solamente favorecen la expansión de los sikhs, sino que permiten su cohesión y constante conexión tanto con el lugar de origen como con la diáspora. Permiten, en definitiva, recrear y mantener los vínculos de la comunidad. Es en este sentido que la comunidad sikh es una comunidad diaspórica (Santos-Fraile 2007) y que sólo puede ser analizada y comprendida desde una amplia y compleja perspectiva como lo es la perspectiva de la globalización, la migración y la diáspora. Pues si bien la migración es una práctica ya arraigada entre los sikhs, el hecho de que haya sikhs en tan gran diversidad de países y los modos en que en ellos se establecen en la actualidad estaría directamente relacionado con esta etapa de la postmodernidad señalada por Appadurai (2001) y que crearía además el contexto que permite que los sikhs continúen con sus estrategias de supervivencia en tanto que grupo y de mejoría socio-económica en tanto que individuos. En el caso de los sikhs, esto es además estimulado y favorecido por unos preceptos religiosos y comunitarios que incentivan la cohesión, la fuerza y su propio potencial, al tiempo como individuos y como comunidad transnacional y virtual.

La comunidad sikh de Barcelona se define por tanto por su adscripción religiosa y un origen $y$ lengua comunes, pero a su vez se caracteriza por su vinculación o pertenencia a una comunidad mucho más global, en tanto que está irremediablemente imbuida de -e imposiblemente ajena a- procesos migratorios y diaspóricos que articulan interrelaciones transnacionales. Si bien su establecimiento en Barcelona está marcado por algunas diferencias sustanciales con respeto a lo observado en los otros lugares de trabajo, es un grupo de establecimiento relativamente reciente y de gran movilidad, que muestra gran versatilidad y capacidad de adaptación a las circunstancias. Esto nos enfrenta a nuevos retos de investigación como sería observar y analizar a otros grupos sikhs establecidos en el Estado Español.

Finalmente, a partir de este trabajo de investigación hemos tenido la oportunidad de conocer y acercarnos a la comunidad sikh de Barcelona, donde sus hombres 
y mujeres destacan por su hospitalidad, generosidad, y riqueza idiosincrásica. Lo que hace deseable, además de pertinente, seguir trabajando sobre esta misma comunidad, observando su evolución y abrir nuevos campos de estudio que se vislumbran de gran interés como son, entre otros, los relacionados con el simbolismo, el rol cambiante de las mujeres más jóvenes o la gestión entre la tradición y el cambio para las nuevas generaciones sikhs.

\section{BIBLIOGRAFÍA CITADA}

Anderson, Benedict. 2000. Comunidades imaginadas. Reflexiones sobre el origen y la difusión del nacionalismo. Buenos Aires: Fondo de cultura Económica.

Appadurai, Arjun. 2001. La modernidad desbordada. Dimensiones culturales de la globalización. Buenos Aires: Fondo de Cultura Económica.

Assad, Talal. 1993. Genealogies of Religion. Baltimore, Md: Johns Hopkins University.

Ballard, Roger y Catherine Ballard. 1979. «The Sikhs: The Development of South Asian Settlements in Britain». En James L. Watson (ed.). Between Two Cultures. Migrants and Minorities in Britain: 21-56. Oxford: Basil Blackwell.

Barth, Fredrik. 1976. «Introduction». En Fredrik Barth (comp.). Ethnic Groups and Boundaries: 9- 38. Long Grove/Illinois: Waveland Press.

Bastos, José. 2010. «Different Children of Different Gods: A Structural-dynamic Approach to Using Religion in Processes of Differentiated Social Insertion». En Charles Westin, José Bastos; Janine Dahinden y Pedro Gois (eds.). Identity Processes and Dynamics in Multi-Ethnic Europe: 279-311. Amsterdam: Amsterdam University Press.

Baumann, Gerd. 2003. Contesting Culture. Discourses of Identity in Multi-Ethnic London. Cambridge: Cambridge University Press.

Benach, Nùria. 2005. «Diferencias e identidades en los espacios urbanos». En Mary Nash, Rosa Telló y Nùria Benach (eds). Inmigración, género y espacios urbanos. Los retos de la diversidad: 71-84. Barcelona: Bellaterra.

Bourdieu, Pierre. 2006. «Préface de Pierre Bourdieu» en Abdelmalek Sayad, L'immigration ou les paradoxes de l'altérité : 9-14. Paris: Éditions Raisons d’Agir.

Brown, Judith M. 2006. Global South Asians. Introducing the Modern Diaspora, Cambridge: Cambridge University Press. doi: <https://doi.org/10.1017/ CBO9780511807657>.

Cole, Owen W. 2004. Understanding Sikhism. Edimburgo: Dunedin Academic Press.

Cole, Owen W. 1994. "Sikhs in Europe». En Sean Gill, Gavin D'Costa y Ursula King (eds.), Religion in Europe: Contemporary Perspectives: 105-119. Kampen, Netherlands: Pharos.
Cole, Owen W. y Piara Singh Sambhi. 2006. The Sikhs. Their Religious Beliefs and Practices. Brighton/Portland: Sussex Academic Press.

Cornejo Valle, Mónica. 2016. "Las definiciones de lo religioso en Antropología Social. Conceptos y discusiones clave en la búsqueda de un universal cultural». Bandue. Revista de la Sociedad Española de Ciencias de las Religiones, 9: 67-88.

Correia, Andre Clareza y Susana Pereira Bastos. 2006. «Quando ela estuda aqui, ela nao faz como eu quero: vulnerabilidades Sikh em Portugal». En Susana Pereira Bastos y Jose Gabriel Pereira Bastos (eds.). Filhos diferentes de deuses diferentes: 179-188. Lisboa: Acime Susana.

García Canclini, Néstor. 2005. Culturas híbridas: estrategias para entrar y salir de la modernidad. Buenos Aires: Paidós.

Godelier, Maurice. 2010. «Community, Society, Culture: Three Keys to Understanding Today's Conflicted Identities». Journal of the Royal Anthropological Institute, 16(1): 1-11. doi: <https://doi.org/10.1111/j.1467-9655.2009.01593.x>.

Herzog, Benno. 2015. «Recognition in Multicultural Societies». Revista Internacional de Sociología, 73 (2). doi: <doi: http:// dx.doi.org/10.3989/ris.2013.07.21>.

Irudaya Rajan, S., V. J Varghese, y Aswini Kumar Nanda. 2015. Migration, Mobility and Multiple Affiliations. Punjabis in a Transnational World. Cambridge: Cambridge University Press.

Jacobsen, Knut A. y Kristina Myrvold. 2011. Sikhs in Europe: Migration, Identities and Representations. Farnham: Ashgate.

Jacobsen, Knut A. y Kristina Myrvold. 2014. Sikhs across Borders: Transnational Practices of European Sikhs. Londres/ Nueva York: Continuum.

Kaur Singh, Nikky-Guninder. 2004. Sikhism. World Religions. Nueva York: Facts on File.

McLeod, Hew. 1997. Sikhism. Londres: Penguin Books.

Marcus, George E. 1998. «Ethnography in/of the World System: The Emergence of Multi-Sited Ethnography». En George E. Marcus, Ethnography through Thick and Thin: 79- 104. Nueva Jersey: Princeton University Press.

Mauss, Marcel. 1979. Sociología y antropología. Madrid: Editorial Tecnos.

Pániker, Agustín. 2007. Los sikhs. Historia, identidad y religión. Barcelona: Kairós.

Provansal, Danielle. 2005. Migraciones y procesos identitarios. Seminario en el marco del Programa de Doctorado en Antropología Social. Universidad de Barcelona, curso 2005/2006. No publicado.

Puar, Jasbir K. 2018. Terrorist Assemblages: Homonationalism in Queer Times. Londres: Duke University Press.

Santos-Fraile, Sandra. 2016. La comunidad sikh de Barcelona. Una aproximación etnográfica. Prácticas, negociación y transformaciones en el cuerpo y la corporalidad tras el proceso migratorio. Tesis doctoral (no publicada). Universitat de Barcelona.

Santos-Fraile, Sandra. 2007. La comunidad Sikh en Barcelona. Una aproximación etnográfica. Trabajo de investigación de 
segundo año del programa del Programa de Estudios Avanzados en Antropologia Social del Departament d)Antropologia Social de la Universitat de Barcelona (no publicado).

Said, Edward W. 2002. Orientalismo. Madrid: Debate.

Sayad, Abdelmalek. 2006. L'immigration ou les paradoxes de l'altérité. Paris: Éditions Raisons d'Agir.

Scheper-Hughes, Nancy. 1999. «Demografía sin números. El contexto económico y cultural de la mortalidad infantil en Brasil». En Andreu Viola, Antropología del desarrollo. Teorías y estudios etnográficos en América Latina: 267-99. Barcelona: Paidós.
Singh Tatla, Darshan. 2004. The Sikh Diaspora. The Search for Statehood. Londres/Nueva York: Routledge.

Thandi, Shinder S. 2014. «Migration and Comparative Experiences of Sikhs in Europe: Reflections on Issues of Cultural Transmission and Identity 30 Years On». En Knut A. Jacobsen y Kristina Myrvold (eds), Sikhs across Borders: Transnational Practices of European Sikhs: 11-35. Londres/Nueva York: Continuum.

Vertovec, Steven. 2009. Transnationalism. Nueva York: Routledge. 